\title{
Model Combination of Activation Functions in Neural Network Algorithms (Case: Retail State Sukuk by Group)
}

\author{
Muhammad Noor Hasan Siregar \\ Universitas Graha Nusantara, Padangsidimpuan, Sumatera Utara \\ noor.siregar@gmail.com
}

\begin{abstract}
This study aims to maximize the activation function used in backpropogation networks in finding the best architectural model. The case study used is the sale of state retail sukuk based on professional groups. The combination of activation functions used for training and testing is tansig-tansig, tansig-purelin and tansig logsig. The architectural model used is the architectural model 6-2-1 and 6-5-1. The evaluation parameters used are epoch, MSE training, MSE testing and accuracy level of truth. Data processing is assisted by using Matlab software. The results showed that the tansig-logsig activation function had more stable results than tansig-tansig and tansig-purelin.
\end{abstract}

Keywords: Activation Function, Artificial Neural Network, Backpropogation, State Retail Sukuk, Combination

\section{Introduction}

Artificial Neural Networks have a positive impact on the progress of current information technology [1]. One method of artificial neural networks used to make predictions is backpropogation [2], [3]. Backpropagation is a type of artificial neural network that uses the supervised learning method used to train ANN until the desired weight is obtained. If the output gives the wrong result, the weigher is corrected so that the error can be minimized and the prediction of the next ANN is expected to be close to the correct value [4]. In this case the process of the activation function to the hidden layer has an effect on the weight learning function on backpropogation [5]. the activation function used must meet the requirements, namely: continuous, easily differentiated and is a function that does not go down. One of the functions that meets these criteria is the binary sigmoid activation function that has a range $(0,1)$. Therefore, this function is often used because it has an output value located in the interval 0-1 [6]. The purpose of this study is to analyze the extent of accuracy of the combination of activation functions in producing a prediction of training and testing data. In this study, the case used was a study conducted by Solikhun et al. 2017 [7] with the topic "Artificial Neural Networks in Predicting Retail State Sukuk Based on Professional Groups with Backpropogation in Encouraging the Rate of Economic Growth". The results of the study mention data from the Ministry of Finance through the website www.djppr.kemenkeu.go.id. The data is the sales data of sukuk with series 001 007. The activation functions used are "logsig" and "tansig". Using the data from previous research [7], the researcher conducted a combination of activation functions where the results of the study will be able to provide maximum information on the use of a series of activation functions. 


\section{Rudimentary}

\subsection{Artificial Neural Network (ANN)}

ANN is compiled by processing elements that are in the related layers and given weights where ANN is an artificial representation of the human brain that always tries to simulate the learning process in the human brain [3].

\subsection{Backpropogation}

ANN is compiled by processing elements that are in the related layers and given weights where ANN is an artificial representation of the human brain that always tries to simulate the learning process in the human brain [2], [5], [7].

\subsection{Activation Function}

The activation function functions to bridge the comparison between the sum of the values of all the weights that will come with the input value with a certain threshold value for each neuron. there are 3 activation functions, namely: (1) linear function (identity / purelin), (2) binary sigmoid function (logsig), (3) sigmoid function bipolar (tansig) [6].

\section{Research Methodology}

The research methodology is the stages in conducting a research to collect data or information that will be used in finding a solution of the problems that have been found.

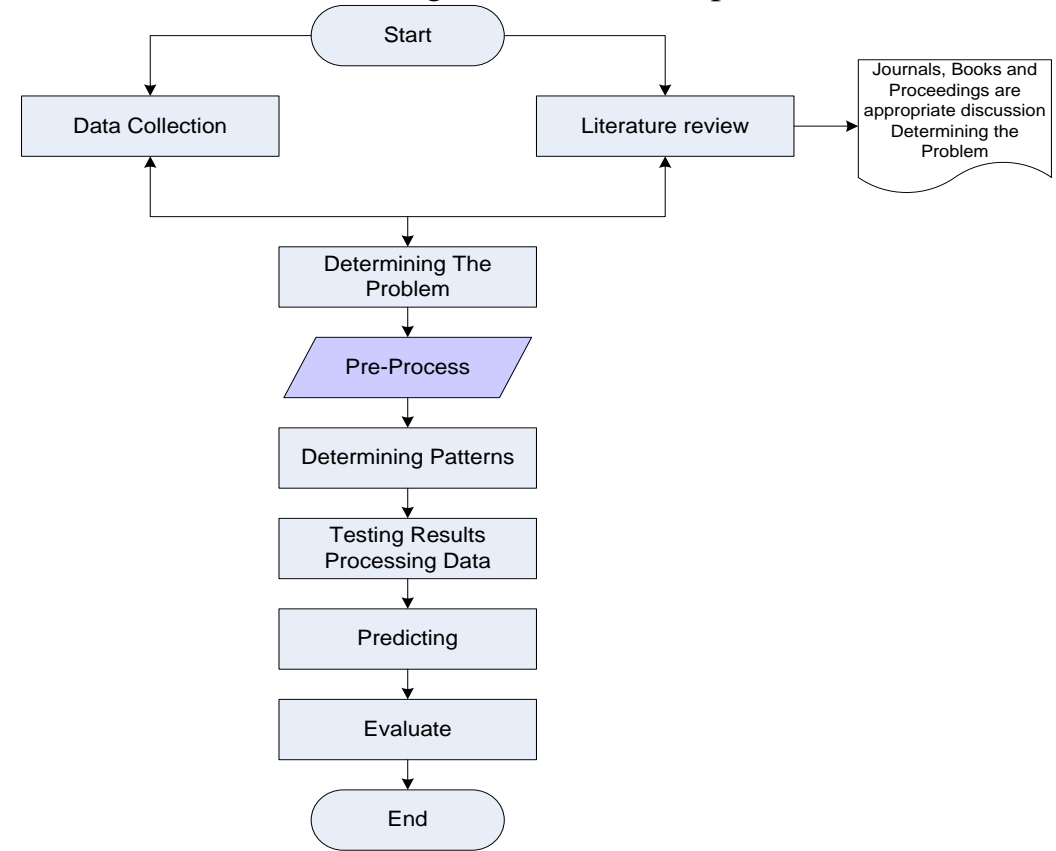

Figure 1. Research Framework

\section{Results and Discussion}

\subsection{Input Data}

In the case: retail state sukuk based on professional groups to determine the best combination of a series of activation functions using the backpropogation algorithm, where data input is a professional group consisting of civil servants (X1), private employees (X2), housewives (X3), entrepreneurs (X4), military / police (X5) and others (X6) [7].

\subsection{Ouput Data}

The output data is the number of state retail sukuk investors based on professional groups [7]. 


\subsection{Data processing}

In this study, the data used to maximize the use of the activation function in the backpropogation algorithm is data from research conducted by Solikhun, et al. (2017) on predictions of state retail sukuk based on professional groups. The process is divided into 2 , namely the training stage and the testing phase. The research data is retail state sukuk data based on professional groups with 7 sales samples from SR001 to SR007 from 2009 to 2016. The activation function used is sigmoid binary (logsig) whose range is from 0 to 1 . So that training data and testing in sukuk cases are obtained state retail based on professional groups such as the following:

Table 1. Research data

\begin{tabular}{|c|c|c|c|c|c|c|c|c|}
\hline \multirow[t]{2}{*}{ No } & \multirow[t]{2}{*}{ Name } & \multicolumn{6}{|c|}{ Variable } & \multirow[t]{2}{*}{ Target } \\
\hline & & $\mathrm{X} 1$ & $\mathrm{X} 2$ & $\mathrm{X} 3$ & $\mathrm{X} 4$ & $\times 5$ & X6 & \\
\hline 1 & SR-001 & 1577 & 5578 & 1560 & 2420 & 40 & 3121 & 14295 \\
\hline 2 & SR-0C & 4099 & 3801 & 3427 & 3274 & 79 & 2550 & 7231 \\
\hline 3 & SR-003 & 3553 & 3677 & 2847 & 2956 & 63 & 2391 & 15487 \\
\hline 4 & SR-004 & 5074 & 3643 & 2849 & 3505 & 56 & 2479 & 17606 \\
\hline 5 & SR-005 & 814 & 5075 & 2956 & 4437 & 350 & 4151 & 17783 \\
\hline 6 & SR-006 & 2713 & 9509 & 5894 & 7934 & 496 & 8146 & 34692 \\
\hline 7 & SR-007 & 2097 & 7629 & 4806 & 8980 & 214 & 5980 & 29706 \\
\hline
\end{tabular}

Table 2. Data that has been transformed

\begin{tabular}{|c|c|c|c|c|c|c|c|c|}
\hline \multirow[t]{2}{*}{ No } & \multirow[t]{2}{*}{ Name } & \multicolumn{6}{|c|}{ Variabel } & \multirow[t]{2}{*}{ Target } \\
\hline & & $\mathrm{X} 1$ & $\mathrm{X} 2$ & X3 & $\mathrm{X} 4$ & $\mathrm{X} 5$ & X6 & \\
\hline 1 & SR-001 & 0,1355 & 0,2279 & 0,1351 & 0,1549 & 0,1000 & 0,1711 & 0,4291 \\
\hline 2 & SR-002 & 0,1937 & 0,1868 & 0,1782 & 0,1747 & 0,1009 & 0,1580 & 0,4969 \\
\hline 3 & SR-003 & 0,1811 & 0,1840 & 0,1648 & 0,1673 & 0,1005 & 0,1543 & 0,4566 \\
\hline 4 & SR-004 & 0,2162 & 0,1832 & 0,1648 & 0,1800 & 0,1004 & 0,1563 & 0,5055 \\
\hline 5 & SR-005 & 0,1179 & 0,2162 & 0,1673 & 0,2015 & 0,1072 & 0,1949 & 0,5096 \\
\hline 6 & SR-006 & 0,1617 & 0,3186 & 0,2352 & 0,2822 & 0,1105 & 0,2871 & 0,9000 \\
\hline 7 & SR-007 & 0,1475 & 0,2752 & 0,2100 & 0,3064 & 0,1040 & 0,2371 & 0,7849 \\
\hline
\end{tabular}

In table 2, it can be explained that the transformation process is based on table 1 because the activation function used is binary $(0-1)$. In this case, training and testing data use the same data. The training process and testing of data are assisted by using the help of Matlab 5.3 software.

\subsection{Combination of Activation Functions}

Before entering the training step and testing artificial neural networks with backpropogation, the authors performed several combinations of activation functions to find the best network architecture model by looking at the error value. The smaller the error value, the better the recommended network architecture model. The following is a combination of activation functions in the case of state retail sukuk based on professional groups.

Table 3. Combination of Activation Functions

\begin{tabular}{|c|l|l|c|}
\hline $\begin{array}{c}\text { Input Combination } \\
\text { Model }\end{array}$ & Type of architecture & Activation Function & $\begin{array}{c}\text { Learning } \\
\text { Function Weight }\end{array}$ \\
\hline Combination 1 & $6-2-1$ and 6-5-1 & Tansig and Logsig & traingd \\
\cline { 3 - 3 } & & Tansig and Purelin & \\
\cline { 2 - 3 } & & Tansig and Tansig & \\
\hline
\end{tabular}


Based on table 3, the training and testing process is carried out using the help of Matlab software with the following parameters:

\begin{tabular}{|c|c|}
\hline code Training & code Testing \\
\hline $\begin{array}{l}\text { > } \text { net }=\text { newff(minmax }(P),[\text { hidden } \\
\text { layer,output layer],\{'Activation Function } 1 \text { ',' } \\
\text { 'Activation Function } 2 '\}, \text { 'traingd'); } \\
>>\text { net.IW }\{1,1\} ; \\
>>\text { net.b }\{1\} ; \\
>\text { net.LW\{2,1\}; } \\
>>\text { net.b }\{2\} ; \\
>\text { net.trainParam.epochs }=2500000 ; \\
>\text { net.trainParam.goal }=0.001 ; \\
>\text { net.trainParam.Lr }=0.1 ; \\
>>\text { net.trainParam.show }=500 ; \\
\text { [a,Pf,Af,e,Perf] }=\operatorname{sim}(\text { net, } P,[],[], T)\end{array}$ & $\begin{array}{l}\text { >PP=[input test data] } \\
>P T=[\text { test output }] \\
{[a, P f, A f, e, P e r f]=\operatorname{sim}(\text { net }, P P,[],[], T T)}\end{array}$ \\
\hline
\end{tabular}

\subsection{Combination Architecture Model 1}

The following are the results of training and testing on the 6-2-1 and 6-5-1 architectural models on combination 1 (according to table 3) seen in the epoch, MSE training, MSE testing and accuracy of truth as shown in the following figure and table:

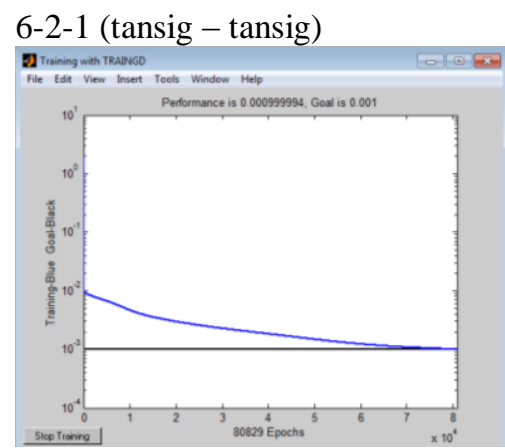

Figure 2. Goal (tansig tansig)

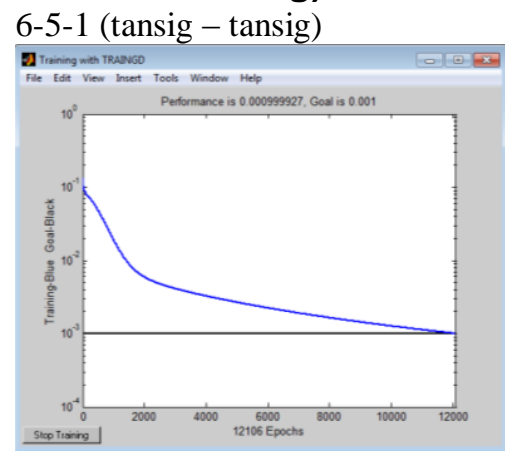

Figure 5. Goal (tansig tansig)

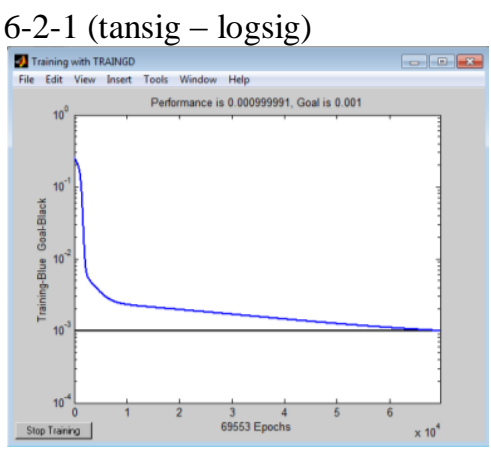

Figure 3. Goal (tansig logsig)

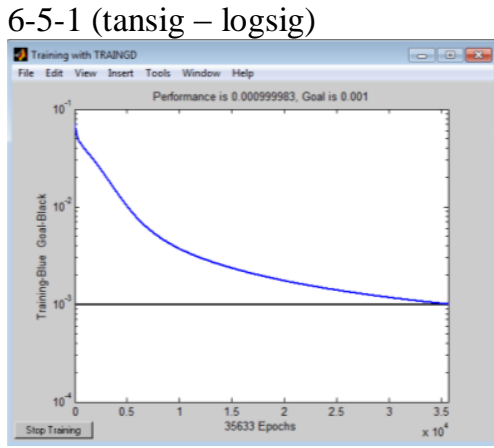

Figure 6. Goal (tansig logsig)

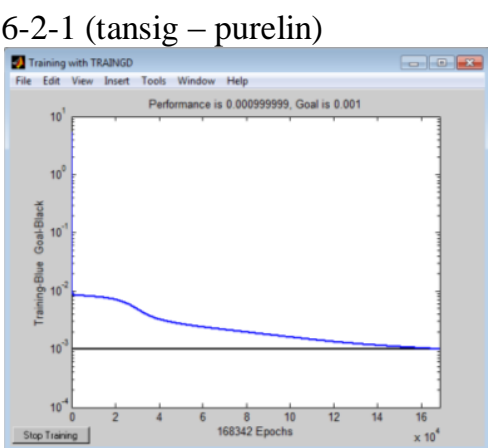

Figure 4. Goal (tansig purelin)

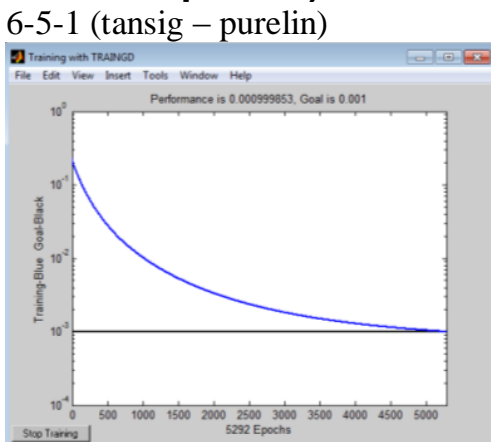

Figure 7. Goal (tansig purelin)

In Figures 2 to 7 , it can be explained that the backpropogation process that achieves goals $=0.001$ based on the activation function with the architectural model 6-2-1 and 6-5-1. The following are the complete results of training and testing data for the architectural model in combination 1 . 
Table 4. Architectural Training and Testing 6-2-1 (tansig - tansig)

\begin{tabular}{|c|c|c|c|c|c|c|c|c|c|}
\hline \multicolumn{10}{|c|}{ Tansig and Tansig } \\
\hline \multicolumn{5}{|c|}{ Training Data } & \multicolumn{5}{|c|}{ Testing Data } \\
\hline No & Target & Output & Error & SSE & No & Target & Output & Error & SSE \\
\hline 1 & 0,4291 & 0,4404 & $-0,0113$ & 0,0001276805 & 1 & 0,4291 & 0,4404 & $-0,0113$ & 0,0001276805 \\
\hline 2 & 0,4969 & 0,5288 & $-0,0319$ & 0,0010186953 & 2 & 0,4969 & 0,5288 & $-0,0319$ & 0,0010186953 \\
\hline 3 & 0,4566 & 0,4201 & 0,0365 & 0,0013336940 & 3 & 0,4566 & 0,4201 & 0,0365 & 0,0013336940 \\
\hline 4 & 0,5055 & 0,5413 & $-0,0358$ & 0,0012787411 & 4 & 0,5055 & 0,5413 & $-0,0358$ & 0,0012787411 \\
\hline 5 & 0,5096 & 0,4985 & 0,0111 & 0,0001238069 & 5 & 0,5096 & 0,4985 & 0,0111 & 0,0001238069 \\
\hline 6 & 0,9000 & 0,9258 & $-0,0258$ & 0,0006656400 & 6 & 0,9000 & 0,9258 & $-0,0258$ & 0,0006656400 \\
\hline 7 & 0,7849 & 0,7353 & 0,0496 & 0,0024591358 & 7 & 0,7849 & 0,7353 & 0,0496 & 0,0024591358 \\
\hline \multicolumn{4}{|c|}{ Total } & 0,0070073937 & \multicolumn{4}{|c|}{ Total } & 0,0070073937 \\
\hline \multicolumn{4}{|c|}{ Means Square Error (MSE) } & 0,0010010562 & \multirow{2}{*}{\multicolumn{4}{|c|}{ Means Square Error (MSE) }} & 0,0010010562 \\
\hline & & & & & & & & & $100 \%$ \\
\hline
\end{tabular}

Table 5. Architectural Training and Testing 6-2-1 (tansig - purelin)

\begin{tabular}{|c|c|c|c|c|c|c|c|c|c|}
\hline \multicolumn{10}{|c|}{ Tansig and Purelin } \\
\hline \multicolumn{5}{|c|}{ Training Data } & \multicolumn{5}{|c|}{ Testing Data } \\
\hline No & Target & Output & Error & SSE & No & Target & Output & Error & SSE \\
\hline 1 & 0,4291 & 0,4307 & $-0,0016$ & 0,0000025587 & 1 & 0,4291 & 0,4307 & $-0,0016$ & 0,0000025587 \\
\hline 2 & 0,4969 & 0,5310 & $-0,0341$ & 0,0011639701 & 2 & 0,4969 & 0,5310 & $-0,0341$ & 0,0011639701 \\
\hline 3 & 0,4566 & 0,4370 & 0,0196 & 0,0003849356 & 3 & 0,4566 & 0,4370 & 0,0196 & 0,0003849356 \\
\hline 4 & 0,5055 & 0,5431 & $-0,0376$ & 0,0014107153 & 4 & 0,5055 & 0,5431 & $-0,0376$ & 0,0014107153 \\
\hline 5 & 0,5096 & 0,4917 & 0,0179 & 0,0003213722 & 5 & 0,5096 & 0,4917 & 0,0179 & 0,0003213722 \\
\hline 6 & 0,9000 & 0,9212 & $-0,0212$ & 0,0004494400 & 6 & 0,9000 & 0,9212 & $-0,0212$ & 0,0004494400 \\
\hline 7 & 0,7849 & 0,7277 & 0,0572 & 0,0032706589 & 7 & 0,7849 & 0,7277 & 0,0572 & 0,0032706589 \\
\hline \multicolumn{4}{|c|}{ Total } & 0,0070036507 & \multicolumn{4}{|c|}{ Total } & 0,0070036507 \\
\hline \multicolumn{4}{|c|}{ Means Square Error (MSE) } & 0,0010005215 & \multirow{2}{*}{\multicolumn{4}{|c|}{$\frac{\text { Means Square Error (MSE) }}{\text { Truth Accuracy }(\%)}$}} & 0,0010005215 \\
\hline & & & & & & & & & $86 \%$ \\
\hline
\end{tabular}

Table 6. Architectural Training and Testing 6-2-1 (tansig - logsig)

\begin{tabular}{|c|c|c|c|c|c|c|c|c|c|}
\hline \multicolumn{10}{|c|}{ Tansig and Logsig } \\
\hline \multicolumn{5}{|c|}{ Training Data } & \multicolumn{5}{|c|}{ Testing Data } \\
\hline No & Target & Output & Error & SSE & No & Target & Output & Error & SSE \\
\hline 1 & 0,4291 & 0,4360 & $-0,0069$ & 0,0000476042 & 1 & 0,4291 & 0,4360 & $-0,0069$ & 0,0000476042 \\
\hline 2 & 0,4969 & 0,5304 & $-0,0335$ & 0,0011233897 & 2 & 0,4969 & 0,5304 & $-0,0335$ & 0,0011233897 \\
\hline 3 & 0,4566 & 0,4307 & 0,0259 & 0,0006718348 & 3 & 0,4566 & 0,4307 & 0,0259 & 0,0006718348 \\
\hline 4 & 0,5055 & 0,5410 & $-0,0355$ & 0,0012573754 & 4 & 0,5055 & 0,5410 & $-0,0355$ & 0,0012573754 \\
\hline 5 & 0,5096 & 0,4911 & 0,0185 & 0,0003432444 & 5 & 0,5096 & 0,4911 & 0,0185 & 0,0003432444 \\
\hline 6 & 0,9000 & 0,9322 & $-0,0322$ & 0,0010368400 & 6 & 0,9000 & 0,9322 & $-0,0322$ & 0,0010368400 \\
\hline 7 & 0,7849 & 0,7347 & 0,0502 & 0,0025190034 & 7 & 0,7849 & 0,7347 & 0,0502 & 0,0025190034 \\
\hline \multicolumn{4}{|c|}{ Total } & 0,0069992920 & \multicolumn{4}{|c|}{ Total } & 0,0069992920 \\
\hline \multicolumn{4}{|c|}{ Means Square Error (MSE) } & 0,0009998989 & \multicolumn{4}{|c|}{ Means Square Error (MSE) } & 0,0009998989 \\
\hline & & & & & \multicolumn{4}{|c|}{ Truth Accuracy (\%) } & $100 \%$ \\
\hline
\end{tabular}

In tables 4 to 6 is the process of training and testing data in the architectural model 62-1 with tansig-tansig activation functions (table 4), tansig-purelin (table 5) and tansiglogsig (table 6). The results obtained are different for each activation function. Evaluation parameters seen from Epoch, MSE training, MSE testing and accuracy level of truth. The following are the complete results of the 6-2-1 architectural model.

\section{Table 7. Recapitulation of Training and Testing data on Architecture 6-2-1}

\begin{tabular}{|l|r|r|r|}
\hline Combination of Activation Functions & tansig-tansig & tansig-purelin & tansig-logsig \\
\hline \multicolumn{4}{|c|}{ Architectural 6-2-1 } \\
\hline Epoch & 80825 & 168342 & 69553 \\
\hline MSE Training & 0,001001056 & 0,001000522 & 0,000999899 \\
\hline MSE Testing & 0,001001056 & 0,001000522 & 0,000999899 \\
\hline Truth Accuracy (\%) & $100 \%$ & $86 \%$ & $100 \%$ \\
\hline
\end{tabular}


Table 8. Architectural Training and Testing 6-5-1 (tansig - tansig)

\begin{tabular}{|r|c|c|c|c|r|c|c|c|c|}
\hline \multicolumn{10}{|c|}{ Tansig and Tansig } \\
\hline \multicolumn{9}{|c|}{} & \multicolumn{7}{|c|}{ Testing Data } \\
\hline No & Target & Output & Error & SSE & No & Target & Output & Error & SSE \\
\hline 1 & 0,4291 & 0,4067 & 0,0224 & 0,0005017788 & 1 & 0,4291 & 0,4067 & 0,0224 & 0,0005017788 \\
\hline 2 & 0,4969 & 0,5519 & $-0,0550$ & 0,0030268709 & 2 & 0,4969 & 0,5519 & $-0,0550$ & 0,0030268709 \\
\hline 3 & 0,4566 & 0,4675 & $-0,0109$ & 0,0001183793 & 3 & 0,4566 & 0,4675 & $-0,0109$ & 0,0001183793 \\
\hline 4 & 0,5055 & 0,4649 & 0,0406 & 0,0016516510 & 4 & 0,5055 & 0,4649 & 0,0406 & 0,0016516510 \\
\hline 5 & 0,5096 & 0,5206 & $-0,0110$ & 0,0001204099 & 5 & 0,5096 & 0,5206 & $-0,0110$ & 0,0001204099 \\
\hline 6 & 0,9000 & 0,8603 & 0,0397 & 0,0015760900 & 6 & 0,9000 & 0,8603 & 0,0397 & 0,0015760900 \\
\hline 7 & 0,7849 & 0,7849 & 0,0000 & 0,0000000001 & 7 & 0,7849 & 0,7849 & 0,0000 & 0,0000000001 \\
\hline \multicolumn{3}{|c|}{ Total } & 0,0069951800 & \multicolumn{5}{c|}{ Total } & 0,0069951800 \\
\hline \multicolumn{3}{|c|}{ Means Square Error (MSE) } & $\mathbf{0 , 0 0 0 9 9 9 3 1 1 4}$ & \multicolumn{7}{c|}{ Means Square Error (MSE) } & $\mathbf{0 , 0 0 0 9 9 9 3 1 1 4}$ \\
\hline
\end{tabular}

Table 9. Architectural Training and Testing 6-5-1 (tansig - purelin)

\begin{tabular}{|c|c|c|c|c|c|c|c|c|c|}
\hline \multicolumn{10}{|c|}{ Tansig and Purelin } \\
\hline \multicolumn{5}{|c|}{ Training Data } & \multicolumn{5}{|c|}{ Testing Data } \\
\hline No & Target & Output & Error & SSE & No & Target & Output & Error & SSE \\
\hline 1 & 0,4291 & 0,4041 & 0,0250 & 0,0006250210 & 1 & 0,4291 & 0,4041 & 0,0250 & 0,0006250210 \\
\hline 2 & 0,4969 & 0,5539 & $-0,0570$ & 0,0032509389 & 2 & 0,4969 & 0,5539 & $-0,0570$ & 0,0032509389 \\
\hline 3 & 0,4566 & 0,4742 & $-0,0176$ & 0,0003090643 & 3 & 0,4566 & 0,4742 & $-0,0176$ & 0,0003090643 \\
\hline 4 & 0,5055 & 0,4552 & 0,0503 & 0,0025341669 & 4 & 0,5055 & 0,4552 & 0,0503 & 0,0025341669 \\
\hline 5 & 0,5096 & 0,5208 & $-0,0112$ & 0,0001248391 & 5 & 0,5096 & 0,5208 & $-0,0112$ & 0,0001248391 \\
\hline 6 & 0,9000 & 0,8901 & 0,0099 & 0,0000980100 & 6 & 0,9000 & 0,8901 & 0,0099 & 0,0000980100 \\
\hline 7 & 0,7849 & 0,7773 & 0,0076 & 0,0000576032 & 7 & 0,7849 & 0,7773 & 0,0076 & 0,0000576032 \\
\hline \multicolumn{4}{|c|}{ Total } & 0,0069996434 & \multicolumn{4}{|c|}{ Total } & 0,0069996434 \\
\hline \multicolumn{4}{|c|}{ Means Square Error (MSE) } & 0,0009999491 & \multicolumn{4}{|c|}{ Means Square Error (MSE) } & 0,0009999491 \\
\hline & & & & & \multicolumn{4}{|c|}{ Truth Accuracy (\%) } & $86 \%$ \\
\hline
\end{tabular}

Table 10. Architectural Training and Testing 6-5-1 (tansig - logsig)

\begin{tabular}{|c|c|c|c|c|c|c|c|c|c|}
\hline \multicolumn{10}{|c|}{ Tansig and Logsig } \\
\hline \multicolumn{5}{|c|}{ Training Data } & \multicolumn{5}{|c|}{ Testing Data } \\
\hline No & Target & Output & Error & SSE & No & Target & Output & Error & SSE \\
\hline 1 & 0,4291 & 0,4302 & $-0,0011$ & 0,0000012091 & 1 & 0,4291 & 0,4302 & $-0,0011$ & 0,0000012091 \\
\hline 2 & 0,4969 & 0,5490 & $-0,0521$ & 0,0027161823 & 2 & 0,4969 & 0,5490 & $-0,0521$ & 0,0027161823 \\
\hline 3 & 0,4566 & 0,4582 & $-0,0016$ & 0,0000024971 & 3 & 0,4566 & 0,4582 & $-0,0016$ & 0,0000024971 \\
\hline 4 & 0,5055 & 0,4444 & 0,0611 & 0,0037381619 & 4 & 0,5055 & 0,4444 & 0,0611 & 0,0037381619 \\
\hline 5 & 0,5096 & 0,5187 & $-0,0091$ & 0,0000823219 & 5 & 0,5096 & 0,5187 & $-0,0091$ & 0,0000823219 \\
\hline 6 & 0,9000 & 0,8784 & 0,0216 & 0,0004665600 & 6 & 0,9000 & 0,8784 & 0,0216 & 0,0004665600 \\
\hline 7 & 0,7849 & 0,7868 & $-0,0019$ & 0,0000036493 & 7 & 0,7849 & 0,7868 & $-0,0019$ & 0,0000036493 \\
\hline \multicolumn{4}{|c|}{ Total } & 0,0070105817 & \multicolumn{4}{|c|}{ Total } & 0,0070105817 \\
\hline \multicolumn{4}{|c|}{ Means Square Error (MSE) } & $\mathbf{0 , 0 0 1 0 0 1 5 1 1 7}$ & \multicolumn{4}{|c|}{ Means Square Error (MSE) } & 0,0010015117 \\
\hline & & & & & \multicolumn{4}{|c|}{ Truth Accuracy (\%) } & $100 \%$ \\
\hline
\end{tabular}

In tables 8 to 10 is the process of training and testing data in the architectural model 65-1 with tansig-tansig activation functions (table 8), tansig-purelin (table 9) and tansiglogsig (table 10). The results obtained are different for each activation function. Evaluation parameters seen from Epoch, MSE training, MSE testing and accuracy level of truth. Calculation process for architectural models 6-5-1 with tansig-tansig, tansig-purelin and tansig-logsig activation functions using matltab software. The following are the complete results of the 6-5-1 architectural model. 
Table 11. Recapitulation of Training and Testing data on Architecture 6-5-1

\begin{tabular}{|l|r|r|r|}
\hline Combination of Activation Functions & tansig-tansig & tansig-purelin & tansig-logsig \\
\hline \multicolumn{4}{|c|}{ Architectural 6-5-1 } \\
\hline Epoch & 12106 & 5292 & 35633 \\
\hline MSE Training & 0,000999311 & 0,000999949 & 0,001001512 \\
\hline MSE Testing & 0,000999311 & 0,000999949 & 0,001001512 \\
\hline Truth Accuracy (\%) & $86 \%$ & $86 \%$ & $100 \%$ \\
\hline
\end{tabular}

\section{Conclusion}

In choosing the best architectural model by combining several activation functions (tansig-tansig, tansig-purelin and tansig-logsig) for architectural models 6-2-1 and 6-5-1 have different results. In the activation function (tansig-purelin), the training process tends to be fast and find goals. This is indicated by the smallest epoch of several activation functions performed. Test results with activation functions (tansig-purelin) tend to be unstable in the prediction process. It can be concluded that a small epoch does not guarantee the results of high predictive accuracy. Likewise with the activation function (tansig-tansig), it has a poor level of accuracy. This hall can be seen from the results of training and testing on the Matlab software trial. For the activation function (tansigtansig), it has a small MSE Training level, but the MSE Test is not stable. Of the three activation functions, the activation function (tansig-logsig) has more stable results seen from training and testing data. Of the 2 architectural models used (6-2-1 and 6-5-1), the activation function (tansig-logsig) is better than the other two activation functions (tansigtansig; tansig-logsig).

\section{References}

[1] A. Sudarsono, "Jaringan Syaraf Tiruan Untuk Memprediksi Laju Pertumbuhan Penduduk Menggunakan Metode Bacpropagation (Studi Kasus Di Kota Bengkulu)," J. Media Infotama, vol. 12, no. 1, pp. 61-69, 2016.

[2] T. Budiharjo, Soemartono, T., Windarto, A.P., Herawan, "Predicting school participation in indonesia using back-propagation algorithm model," Int. J. Control Autom., 2018.

[3] T. Budiharjo, Soemartono, T., Windarto, A.P., Herawan, "Predicting tuition fee payment problem using backpropagation neural network model," Int. J. Adv. Sci. Technol., 2018.

[4] R. Adistya and M. A. Muslim, "Deteksi dan Klasifikasi Kendaraan menggunakan Algoritma Backpropagation dan Sobel," J. Mech. Eng. Mechatronics, vol. 1, no. 2, pp. $65-73,2016$.

[5] A. P. Windarto, M. R. Lubis, and Solikhun, "Implementasi JST Pada Prediksi Total Laba Rugi Komprehensif Bank Umum Konvensional Dengan Backpropagation," J. Teknol. Inf. dan Ilmu Komput., vol. 5, no. 4, pp. 411-418, 2018.

[6] A. Trimulya, Syaifurrahman, and F. A. Setyaningsih, "Implementasi jaringan syaraf tiruan metode backpropagation untuk memprediksi harga saham," $J$. Coding, Sist. Komput. Untan, vol. 3, no. 2, pp. 66-75, 2015.

[7] Solikhun, A. P. Windarto, Handrizal, and M.Fauzan, "Jaringan Saraf Tiruan Dalam Memprediksi Sukuk Negara Ritel Berdasarkan Kelompok Profesi Dengan Backpropogation Dalam Mendorong Laju Pertumbuhan Ekonomi," Kumpul. J. Ilmu Komput., vol. 4, no. 2, pp. 184-197, 2017. 


\section{Authors}

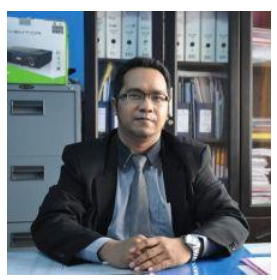

$1^{\text {st }}$ Author

Muhammad Noor Hasan

Universitas Graha Nusantara, Padangsidimpuan

noor.siregar@gmail.com 